\title{
A cinnamyl alcohol dehydrogenase required for sclerotial development in Sclerotinia sclerotiorum
}

\author{
Jiahong Zhou ${ }^{1,2+}$, Yang Lin ${ }^{2+}$, Yanping Fu², Jiatao Xie ${ }^{2}$, Daohong Jiang ${ }^{1,2}$ and Jiasen Cheng ${ }^{1,2^{*}}$ (D)
}

\begin{abstract}
Sclerotial development is a vital stage in the life cycles of many fungal plant pathogens. In this study, the protein Ss-CAD, which contains three conserved domains of cinnamyl alcohol dehydrogenase (CAD), was found to be required for sclerotial development in Sclerotinia sclerotiorum. Ss-CAD was significantly upregulated during early stage of sclerotial development. In Ss-CAD-silenced strains, sclerotial development was abnormal. In these silenced strains, formation of sclerotia was delayed or sclerotia yield was reduced, whereas hyphal growth and virulence were normal. Nox1, Nox2, and NoxR, which encode reactive oxygen species (ROS)-generating NADPH oxidases, were downregulated in Ss-CAD-silenced strains. NoxR-silenced strains displayed similar defects during sclerotial formation as Ss-CAD-silenced strains. Treatment of SS-CAD-silenced strains with exogenous oxidants or NADPH restored normal sclerotial development. Sclerogenesis in Ss-CAD-silenced strains could also be recovered through Nox 1 overexpression. The results suggest that Ss-CAD is linked to the NADPH oxidase pathways to affect sclerotial development in S. sclerotiorum.
\end{abstract}

Keywords: Cinnamyl alcohol dehydrogenase, NADPH, Sclerotial development, Sclerotinia sclerotiorum

\section{Background}

Sclerotinia sclerotiorum (Lib.) de Bary is a globally distributed necrotrophic fungal pathogen. It infects more than 450 plant species across 75 families and causes significant yield losses in oilseeds such as rape, sunflower, and soybean (Boland and Hall 1994; Hegedus and Rimmer 2005; Bolton et al. 2006). The sclerotia of S. sclerotiorum can survive in the soil for more than 8 years and protect this fungus against low temperatures, low moisture, high UV, and microbial activity (Bolton et al. 2006). When the environmental conditions are appropriate, the sclerotia germinate either myceliogenically to form hyphae or carpogenically to release copious ascospores,

\footnotetext{
* Correspondence: jiasencheng@mail.hzau.edu.cn

${ }^{\dagger}$ Jiahong Zhou and Yang Lin contributed equally to this work.

'National Key Laboratory of Agriculture Microbiology, Huazhong Agricultural University, Wuhan 430070, Hubei Province, P.R. China

${ }^{2}$ The Provincial Key Lab of Plant Pathology of Hubei Province, College of Plant Science and Technology, Huazhong Agricultural University, Wuhan
} 430070, Hubei Province, P.R. China which inoculate host plants and induce disease. Forming new sclerotia enables $S$. sclerotiorum to survive under adverse environmental conditions (Adams 1979; Steadman 1979; Roper et al. 2010). As sclerotial development is vital in the S. sclerotiorum disease cycle, blocking this process may effectively prevent Sclerotinia diseases in the field.

Sclerotial development is complex and artificially divided into six sequential stages-initiation, condensation, enlargement, consolidation, pigmentation, and maturation (Li and Rollins 2009). An earlier study demonstrated that several environmental factors, such as nutrition, light, $\mathrm{pH}$, temperature, and oxidative stress, are associated with sclerotial development (Chet and Henis 1975). Numerous recent studies focusing on the molecular mechanisms underlying sclerotial development have revealed that several genes regulate the process (Chen et al. 2004; Jurick et al. 2004; Chen and Dickman 2005; Harel et al. 2006; Erental et al. 2007;

(c) The Author(s). 2020 Open Access This article is licensed under a Creative Commons Attribution 4.0 International License, which permits use, sharing, adaptation, distribution and reproduction in any medium or format, as long as you give appropriate credit to the original author(s) and the source, provide a link to the Creative Commons licence, and indicate if changes were made. The images or other third party material in this article are included in the article's Creative Commons licence, unless indicated otherwise in a credit line to the material. If material is not included in the article's Creative Commons licence and your intended use is not permitted by statutory regulation or exceeds the permitted use, you will need to obtain permission directly from the copyright holder. To view a copy of this licence, visit http://creativecommons.org/licenses/by/4.0/. 
Jurick and Rollins 2007; Li and Rollins 2010; Kim et al. 2011; Fan et al. 2017; Li et al. 2018; Liang et al. 2018; Zhang et al. 2019a). We also reported that Ss-Sl2, SSITL, Ss-Caf1, and Sop1 were associated with sclerotial development in S. sclerotiorum (Yu et al. 2012; Zhu et al. 2013; Xiao et al. 2014; Lyu et al. 2016). S. sclerotiorum coordinates environmental signals and triggers the mitogen-activated protein kinase (MAPK) signaling pathway mediated by $S m k 1$, which is a downstream ERK-type MAPK. In turn, this promotes the formation of sclerotia. Increase in levels of cAMP may result in defective sclerogenesis as cAMP inhibits the MAPK signaling pathway. Rap-1, a small GTPase, is activated by cAMP and binds to an unidentified MAPK kinase kinase (MAPKKK). Hence, MAPKKK is separated from Ras, Smk1 is inactivated, and sclerogenesis is suppressed (Chen et al. 2004; Jurick et al. 2004; Chen and Dickman 2005). Several genes associated with oxidative stress may regulate sclerotial differentiation in S. sclerotiorum. Silencing the SsNox1 and SsNox2 NADPH oxidase genes reduces ROS levels and inhibits sclerotial development (Kim et al. 2011). The Sop1 microbial opsin homolog negatively regulates $S$. sclerotiorum resistance to oxidative stress and is essential for sclerotial development (Lyu et al. 2016). Sstrr1 encoding a thioredoxin reductase (TrxR), a critical enzyme for maintenance of cellular redox homeostasis, is associated with sclerogenesis. Sstrr1-silenced strains have been shown to produce relatively fewer but larger sclerotia (Zhang et al. 2019a). However, understanding of the molecular mechanisms underlying sclerotial development is lacking and these mechanisms remain to be elucidated.

The presence of cinnamyl alcohol dehydrogenase (CAD, EC 1.1.1.195) was first biochemically established in soybean (Glycine max) (Ebel and Grisebach 1973), and the enzyme is ubiquitous in plants. In fact, numerous plant species possess a family of CAD-like genes (Kim et al. 2004; Tobias and Chow 2005; Saballos et al. 2009; Preisner et al. 2014). CADs contain a highly conserved Rossmann fold NAD $(\mathrm{P}) \mathrm{H} / \mathrm{NAD}(\mathrm{P})+$ binding domain and are NADPH- and $\mathrm{Zn}^{2+}$-dependent (Rossmann et al. 1974; Jornvall and Hoog 1995). In addition, CADs use NADPH as a cofactor and catalyze the reversible conversion of $p$-hydroxycinnamaldehydes to their corresponding alcohols. This reaction is the final step of monolignol biosynthesis in plants and mushrooms (Boerjan et al. 2003; Kim et al. 2004; Li et al. 2016). However, certain CAD homologs in bacteria and yeast are not associated with lignin biosynthesis (Wilkin et al. 1999; Larroy et al. 2002; Valencia et al. 2004; Seo et al. 2012). Genome sequence databases indicate that many filamentous fungi also encode CAD homologs. However, their biochemical functions in fungi have not yet been determined.
We previously constructed gene expression profiles during hyphal growth and sclerotial development using digital gene expression (DGE, Solexa/Illumina) technology based on deep sequencing (Lyu et al. 2015). The expression profile database (GEO accession No. GSE65301 for the DGE data) revealed that several genes were significantly upregulated or downregulated during sclerotial development. Thus, these genes might play important roles in the process. A gene with highly conserved CAD motifs (SS1G_10803; GenBank accession No. XM_001588306.1), designated Ss-CAD, was upregulated more than 100-times during sclerotial development. Therefore, the present study endeavored to determine the role of Ss-CAD in sclerotial development.

\section{Results}

\section{Ss-CAD contains conserved CAD domains}

It was predicted that $S s-C A D$ would encode a protein composed of 333 amino acids. The molecular weight and isoelectric points of Ss-CAD were near $36.15 \mathrm{kD}$ and 6.23, respectively. It had previously been established that a typical CAD has a catalytic $\mathrm{Zn} 1$ binding motif $\left[\mathrm{GHE}(\mathrm{X})_{2} \mathrm{G}(\mathrm{X})_{5} \mathrm{G}(\mathrm{X})_{2} \mathrm{~V}\right]$, a structural $\mathrm{Zn} 2$ binding motif $\left[\mathrm{GD}(\mathrm{X})_{9.10} \mathrm{C}(\mathrm{X})_{2} \mathrm{C}(\mathrm{X})_{2} \mathrm{C}(\mathrm{X})_{7} \mathrm{C}\right]$, and an $\mathrm{NADPH}$ binding motif $[\mathrm{G}(\mathrm{X}) \mathrm{GGV}(\mathrm{L}) \mathrm{G}]$, and that all three are highly conserved (McKie et al. 1993). Through motif analysis using MEME SUITE, it was found that these three highly conserved motifs also existed in Ss-CAD (Bailey et al. 2009; Additional file 1: Figure S1).

\section{Ss-CAD is highly expressed at the initiation stage of sclerotial development and its downregulation impairs this process}

Quantitative real-time reverse-transcription PCR (qRTPCR) was performed to identify $S s-C A D$ transcript profiles at various stages of mycelial growth on potato dextrose agar (PDA). Ss-CAD expression peaked on the third day after inoculation, at which point sclerotial development began. At this point, Ss-CAD expression was approximately 130 -fold higher than that at the vegetative growth stage, which occurred on the second day after inoculation. Thereafter, $S s-C A D$ expression gradually declined (Fig. 1a). As sclerotiogenesis begins 3-4 d after inoculation onto PDA ( $\mathrm{Li}$ and Rollins 2009; Yu et al. 2012), it can be inferred that $S s-C A D$ participates in $S$. sclerotiorum sclerotial development. To test this hypothesis, Ss-CAD was silenced by RNA interference (RNAi). A gene-silencing vector was constructed and transformed into the wild-type strain EP-1PNA367. After hyphal tip purification, 98 independent transformants were obtained, which were confirmed by PCR screening of the selectable marker, the hygromycin resistance gene $h p h$. qRT-PCR was then performed to detect $S s-C A D$ expression level in the transformants. Ss-CAD expression and CAD activity were both significantly reduced in the 
a

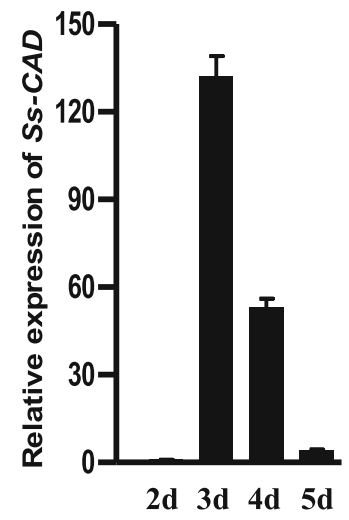

b

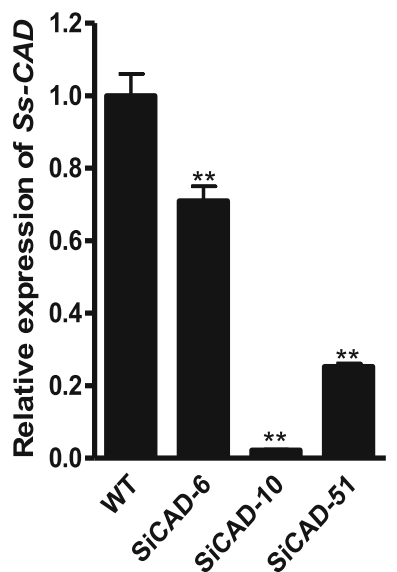

C

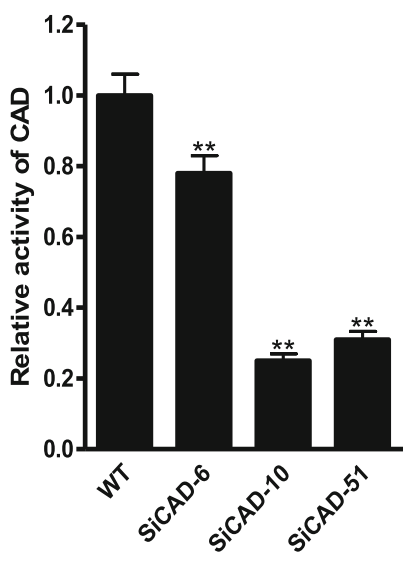

Fig. 1 Ss-CAD-silenced transformants showing reduced Ss-CAD expression levels and CAD activity. a Ss-CAD CDNA expression levels were measured by qRT-PCR and were normalized to that of actin CDNA in extracts taken from each developmental stage. CDNA abundances from samples grown on PDA for $2 \mathrm{~d}$ were assigned a value of 1.0. For all experiments, three independent replicates were performed. $\mathbf{b}$ qRT-PCR analysis of Ss-CAD transcript accumulation in Ss-CAD-silenced transformants. Ss-CAD expression in the wild-type strain Ep-1PNA367 served as control. Hyphal masses from 3-day-old colonies on PDA were collected for gene expression analysis. c CAD activity in the wild-type strain and SsCAD-silenced transformants was measured by enzyme activity assays. CAD activity in the wild-type strain was assigned a value of 1.0. Three independent replicates were performed for all experiments. Values are means \pm S.E. ${ }^{* *} P<0.01$; one-way ANOVA

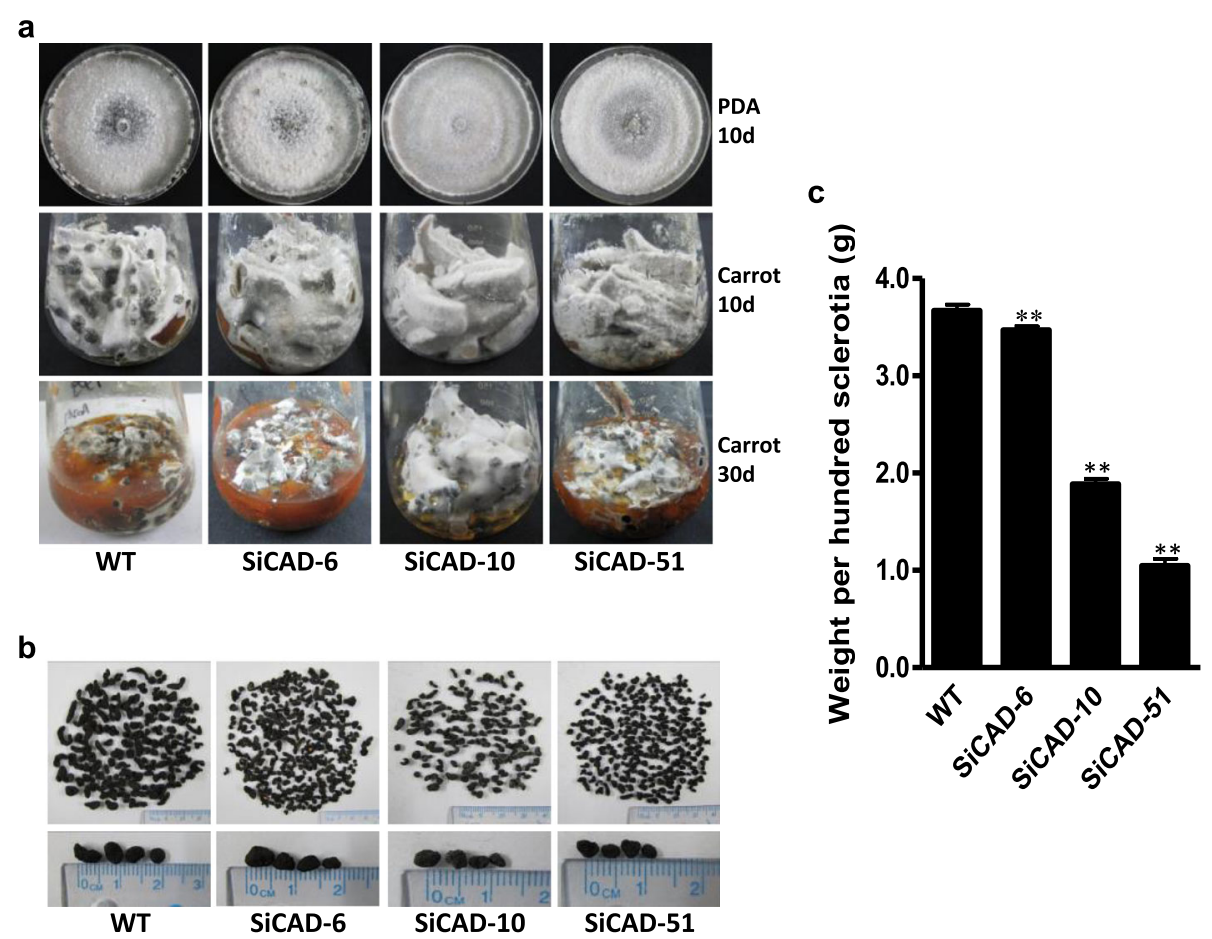

Fig. 2 Ss-CAD-silenced transformants showing abnormal sclerotial development. a Phenotypes of the wild-type strain and Ss-CAD gene-silenced transformants were cultured on PDA and carrots. Photographs were taken 10 and 30 days post-inoculation (dpi). b Relative sizes of sclerotia formed by Ss-CAD-silenced transformants and the wild-type strain on carrot rods. c Relative weights of sclerotia formed by Ss-CAD-silenced transformants and the wild-type strain on carrot rods. Sclerotia were produced on autoclaved carrot rods incubated in $250-\mathrm{mL}$ flasks at $20^{\circ} \mathrm{C}$ for $30 \mathrm{~d}$. For each strain, 100 randomly selected sclerotia were photographed and weighed. Three independent replicates were performed for all experiments. Values are means \pm S.E. ${ }^{* *} P<0.01$; one-way ANOVA 
SiCAD-10 and SiCAD-51 transformants (Fig. 1b, c). Hence, these transformants were selected for further study. The transformant SiCAD-6, in which Ss-CAD expression and CAD activity were both slightly decreased, was also analyzed.

Neither fungal vegetative growth rate on PDA nor virulence on detached rapeseed leaf differed markedly between the SiCAD-10 and SiCAD-51 transformants and the wild-type strain (Additional file 2: Figure S2). However, the initiation of sclerotial development was delayed by $3-4 \mathrm{~d}$ in these transformants compared to that in the wild-type strain. Sclerotia were only observed in the wild-type strain after $10 \mathrm{~d}$ of incubation on PDA or carrot, at which point no sclerotia were visible in either SiCAD-10 or SiCAD-51 (Fig. 2a). All strains eventually produce mature sclerotia. On the contrary, the weights of 100 sclerotia of SiCAD-10 and SiCAD-51 were nearly $50 \%$ less than that of the wild-type strain (Fig. 2b, c). These results suggest that $S S-C A D$ is involved in sclerotial development and that $S S-C A D$ silencing delays sclerogenesis and reduces sclerotia formation.

\section{Superoxide production is impaired in Ss-CAD-silenced strains}

A previous study demonstrated that NADPH oxidases regulate sclerotial development in S. sclerotiorum (Kim et al. 2011). Ss-Nox2 silencing impeded sclerotial development but did not reduce pathogenicity relative to that in the wild-type strain, and produced a phenotype resembling that of $S s-C A D$-silenced strains. Since the superoxide level in Ss-Nox2-silenced strains was reduced at the initiation stage of sclerotial development, we wondered whether a similar phenomenon would occur in Ss$C A D$-silenced strains. Superoxide was then detected by Nitro blue tetrazolium (NBT) staining at the early stage of sclerotial development in both the wild-type and Ss$C A D$-silenced strains. Dark blue formazan precipitates were observed in the wild-type strain, whereas superoxide accumulation was significantly reduced in SiCAD10 and SiCAD-51 (Fig. 3a).

\section{Exogenous oxidants and NADPH treatment restore sclerotial development in Ss-CAD-silenced transformants} The $S s$-CAD-silenced strain was incubated for $15 \mathrm{~d}$ on PDA modified with $6 \mathrm{mM} \mathrm{H}_{2} \mathrm{O}_{2}$ to confirm that $S s-C A D$ influences sclerotial development via the reactive oxygen species (ROS) pathway. This treatment augmented sclerotial development in the wild-type and SiCAD-51 strains. In fact, sclerotia production in SiCAD-51 was partially recovered. Moreover, 0.2 M NADPH completely restored sclerotia biogenesis in SiCAD-51 (Fig. 3b, c). Therefore, superoxide production was impaired in Ss-

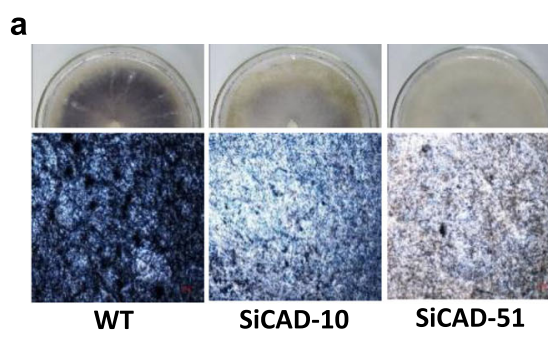

b

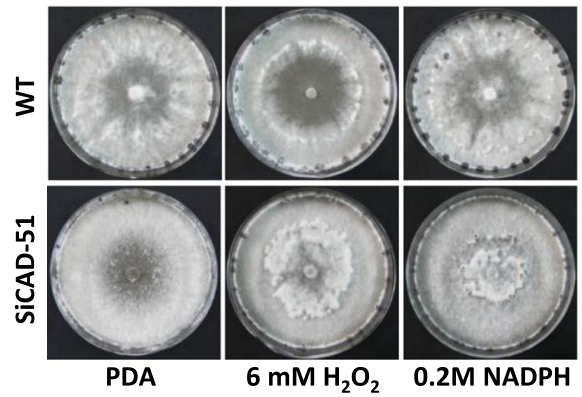

C

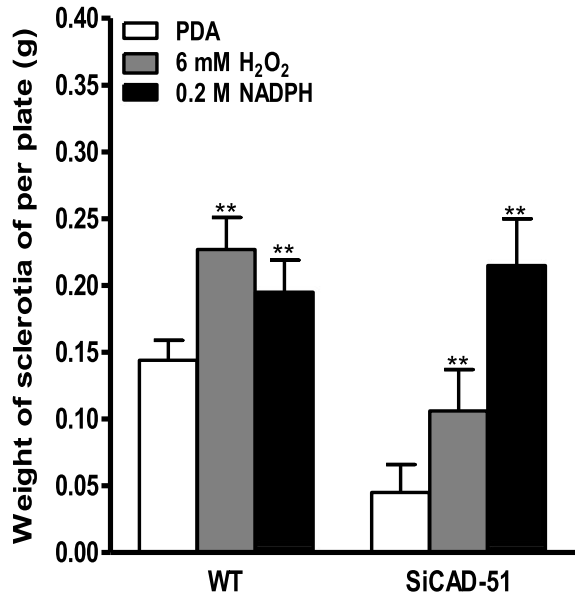

WT
SiCAD-51

Fig. $3 \mathrm{Ss}$-CAD-silenced transformants showing reduced superoxide accumulation. Exogenous $\mathrm{H}_{2} \mathrm{O}_{2}$ and NADPH treatments restored sclerotial development in Ss-CAD-silenced transformants. a NBT staining was used to detect superoxide accumulation at 3-4 dpi on PDA. Staining intensity was monitored under a light microscope, and blue staining represented superoxide accumulation. Ss-CAD-silenced transformants accumulated less blue formazan than the wild-type strain EP-1PNA367. $\mathbf{b}$ Treatment with exogenous $\mathrm{H}_{2} \mathrm{O}_{2}$ and NADPH recovered sclerotial development in SsCAD-silenced transformants. The effects of adding $6 \mathrm{mM} \mathrm{H}_{2} \mathrm{O}_{2}$ and $0.2 \mathrm{mM} \mathrm{NADPH}$ individually to PDA on S. sclerotiorum sclerotial development were evaluated. Photographs were taken at $10 \mathrm{dpi}$. c Weights of sclerotia formed under various culture conditions. Sclerotia produced on each plate were collected, air-dried, and weighed at $15 \mathrm{dpi}$. Three independent replicates were performed for each experiment. Values are means \pm S.E. ${ }^{* *} P<0.01$; one-way ANOVA 
$C A D$-silenced strains. Further, $S s-C A D$ might be linked to the NADPH oxidase pathway and regulate sclerotial development in S. sclerotiorum.

\section{Ss-CAD silencing downregulates ROS-generating genes}

To clarify the relationship between $S s-C A D$ silencing and ROS reduction, the expression levels of the Ss-Nox1, Ss-Nox2, and Ss-NoxR ROS-generating NADPH oxidase genes were detected via qRT-PCR. The expression levels of these genes peaked at the start of sclerotial development and gradually declined thereafter. This trend resembled that of Ss-CAD expression levels (Fig. 4a). Significant reductions in the expression levels of these genes were also observed in SiCAD-10 and SiCAD-51 compared to those in the wild-type strain (Fig. 4b). Thus, Ss-CAD participates in interactions with the NADPH oxidase pathway in S. sclerotiorum.

\section{Ss-NoxR silencing impairs formation of sclerotia}

Previous studies have shown that NoxR plays a key role in fungal ROS production (Takemoto et al. 2006; Scott and Eaton 2008; Tanaka et al. 2008; Hernandez-Onate et al. 2012). The present study found that the expression pattern of Ss-NoxR was similar to that of Ss-CAD and peaked at the start of sclerotial development, which implied that Ss-NoxR might also play an important role in sclerotial development in S. sclerotiorum. Therefore, the SiNoxR-8 and SiNoxR-20 Ss-NoxR-silenced transformants were created. qRT-PCR showed that, compared to the wild-type strain, the number of Ss-NoxR transcripts was significantly reduced in SiNoxR-8 and SiNoxR-20 (Fig. 5a). These strains displayed defective sclerotial development and produced highly branched hyphae, resembling the phenotype of the Ss-Nox2 mutant (Fig. 5b, c) (Kim et al. 2011). Therefore, ROS generation positively regulates sclerotial development in $S$. sclerotiorum. a

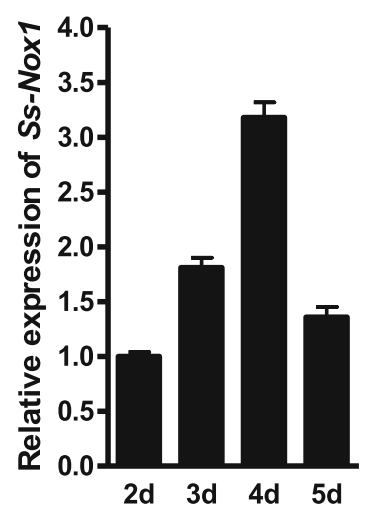

b

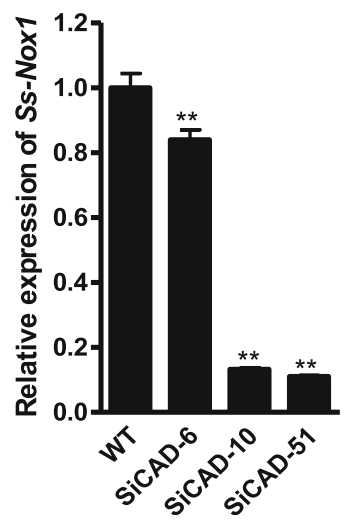

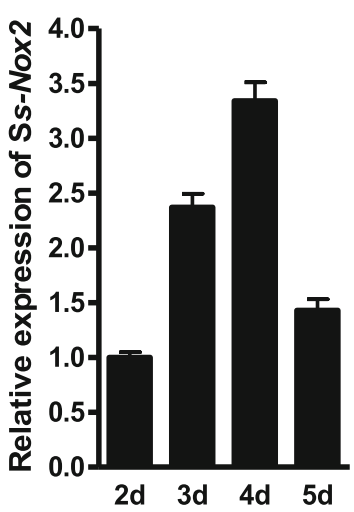
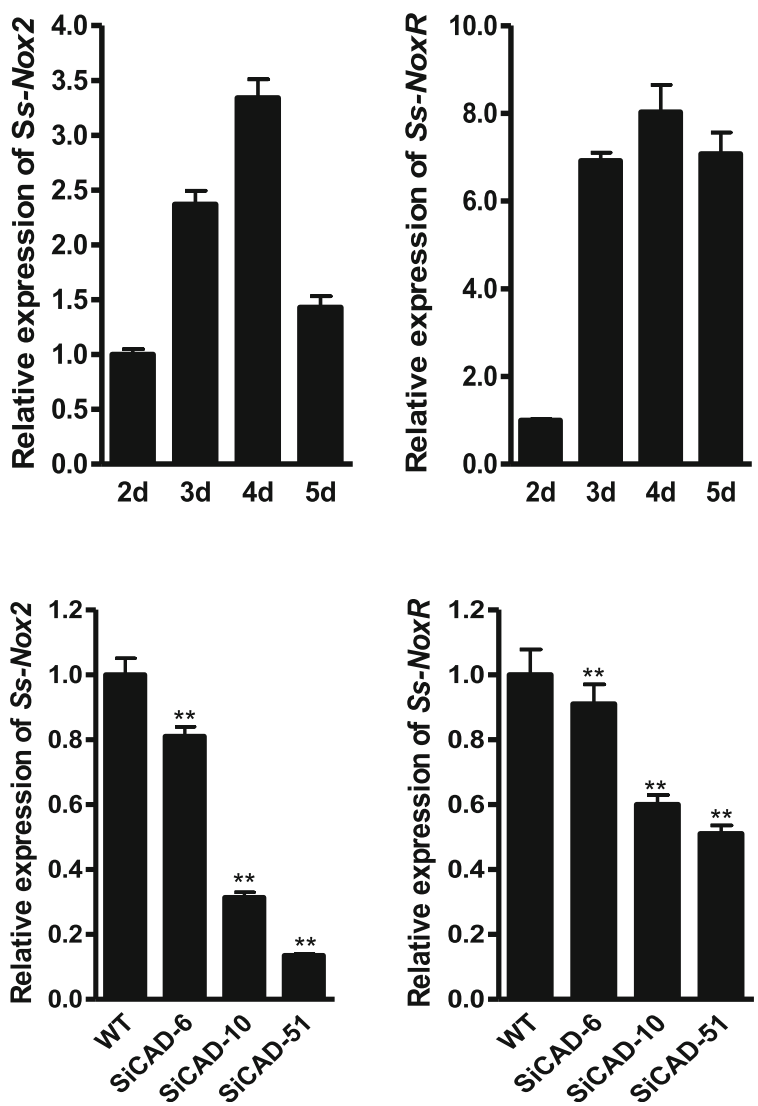

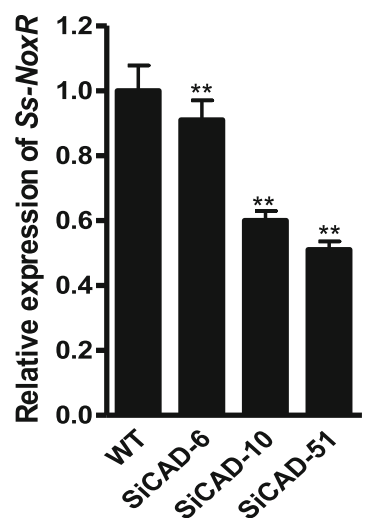

Fig. 4 Expression levels of Ss-Nox1 (GenBank accession No. XP_001592740), Ss-Nox2 (GenBank accession No. XP_001587930), and Ss-NoxR (GenBank accession No. XP_001598015) in the wild-type strain EP-1PNA367 and Ss-CAD-silenced transformants. a qRT-PCR analysis of Ss-Nox1, SsNox2, and Ss-NoxR transcript levels at different sclerotial development stages in the wild-type strain. $\mathbf{b}$ qRT-PCR analysis of Ss-Nox1, Ss-Nox2, and Ss-NoxR transcript levels in Ss-CAD-silenced transformants. Ss-Nox1, Ss-Nox2, and Ss-NoxR cDNA expression levels were measured using qRT-PCR and were normalized to actin cDNA. Three independent replicates were performed for each experiment. Values are means $\pm S$.E. ${ }^{* *} P<0.01$; one-way ANOVA 


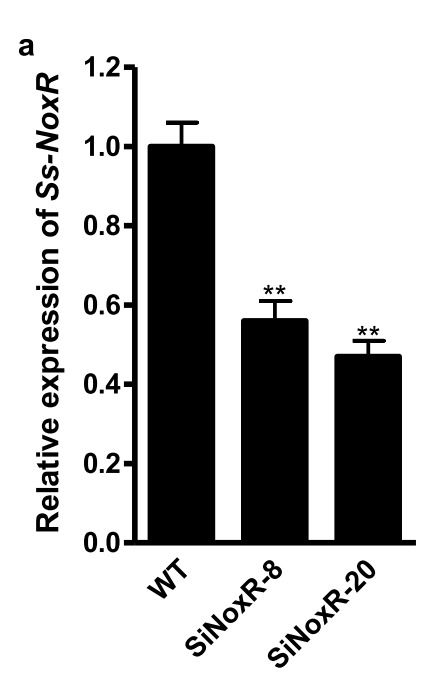

b
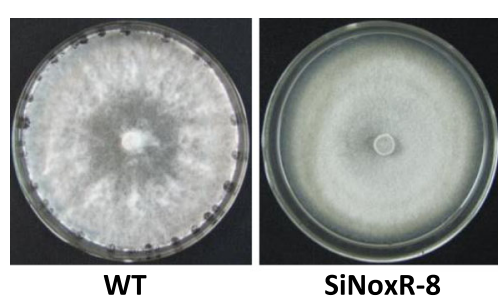

SiNoxR-8

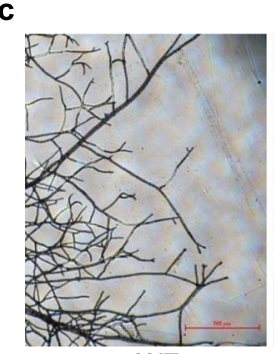

WT

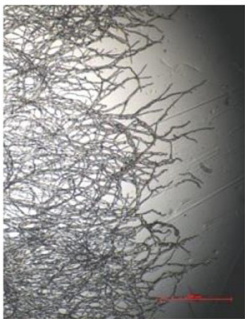

SiNoxR-8

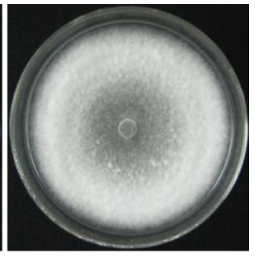

SiNoxR-20

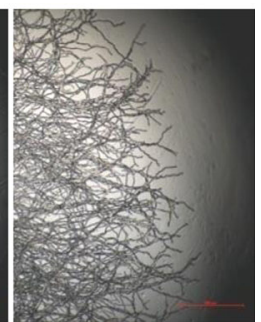

SiNoxR-20

Fig. 5 Ss-NoxR-silenced transformants lost the ability to form sclerotia. a qRT-PCR analysis of Ss-NoxR transcript accumulation in Ss-NoxR-silenced transformants. Ss-NoxR expression in the wild-type strain Ep-1PNA367 served as control. Hyphal masses from 3-day-old colonies on PDA were collected for gene expression analysis. The cDNA abundance obtained from the wild-type strain was assigned a value of 1.0. Three independent replicates were performed. Values are means \pm S.E. ${ }^{* *} P<0.01$; one-way ANOVA. $\mathbf{b}$ Phenotypes of the wild-type strain EP-1PNA367 and Ss-NoxRsilenced transformants incubated on PDA for $10 \mathrm{~d}$. c In vitro hyphal development of the wild-type strain and Ss-NoxR-silenced transformants. All strains were cultured on PDA for $1 \mathrm{~d}$. Hyphal tips were observed under a dissecting microscope. Bars $=500 \mu \mathrm{m}$

\section{Nox1 overexpression restores sclerotial development in} Ss-CAD-silenced strains

The Ss-Nox1 overexpression vector was constructed using the G418 resistance gene, and the vector was then transformed into the $S s$-CAD-silenced strain SiCAD-51 to discern the relationship between SsCAD and NADPH oxidases, as well as their roles in sclerotial development. This resulted in the SiCADOE68 and SiCAD-OE77 overexpression strains. qRTPCR analysis confirmed that SsNox 1 transcription was entirely recovered in SiCAD-OE68 and SiCAD-OE77 (Fig. 6a). Moreover, sclerotia initiation and the numbers of sclerotia formed were restored to the levels of the wild-type strain (Fig. 6b). Hence, Ss-CAD directly

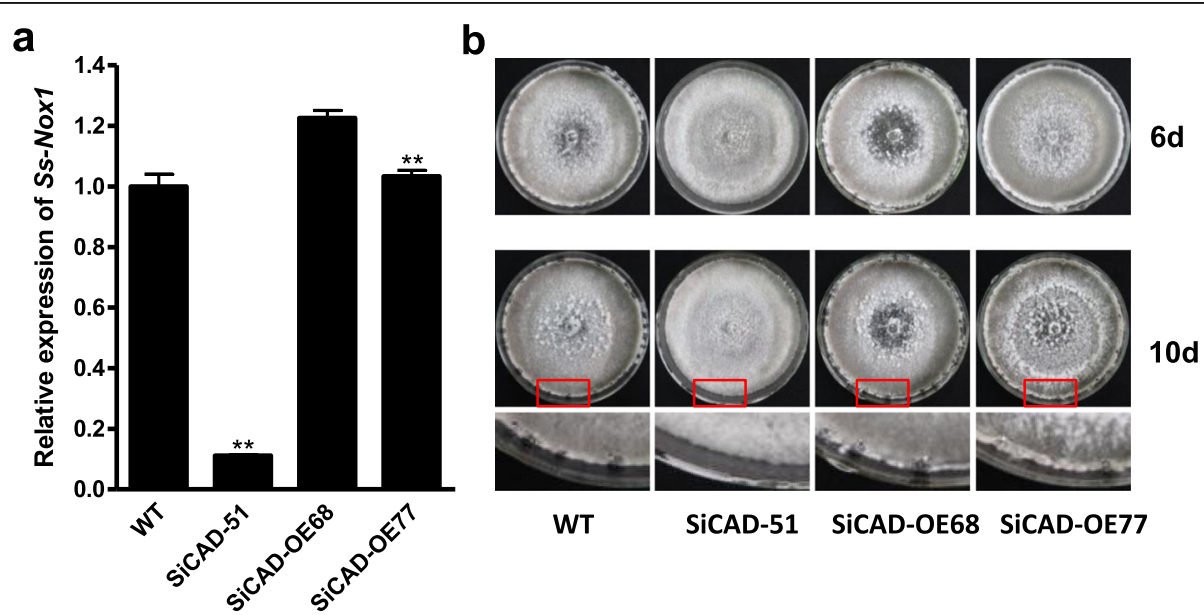

Fig. 6 Ss-Nox1 overexpression restored sclerotial development in Ss-CAD-silenced transformants. a qRT-PCR analysis of Ss-nox1 transcript accumulation. The CDNA abundance obtained from the wild-type strain was assigned a value of 1.0. Three independent replicates were performed. Values are means \pm S.E. ${ }^{* *} P<0.01$; one-way ANOVA. b Sclerotial development in the SiCAD-OE68 and SiCAD-OE77 SS-Nox1overexpressing strains on PDA. Sclerotial development was defective in SiCAD-51 and was restored in Ss-Nox1-overexpressing strains. Photographs were taken at $6 \mathrm{dpi}$ and at $10 \mathrm{dpi}$ 
or indirectly regulates sclerotial development in $S$. sclerotiorum via the ROS pathway.

\section{Discussion}

Sclerotial development is a vital stage of the S. sclerotiorum life cycle. This study examined how the Ss-CAD gene regulates sclerotial development in S. sclerotiorum. At the initiation stage of sclerotial development, Ss-CAD was significantly upregulated. In contrast, the expression levels of other S. sclerotiorum CAD homologs were very low at this stage. $S s-C A D$ silencing delayed and reduced sclerotial development, and there was no evidence that Ss-CAD participates in S. sclerotiorum pathogenicity as its silencing had no apparent effect on fungal virulence.

CADs were first identified in plants, and their homologs were subsequently detected in bacteria and yeast (Ebel and Grisebach 1973; Larroy et al. 2002; Seo et al. 2012). In plants, CADs catalyze the biosynthesis of three different monolignols, thus determining lignin content and composition (Sibout et al. 2005). The phenylpropanoid biosynthetic pathway governs lignification and generates numerous phenolic derivatives such as flavonols, anthocyanins, sinapate esters, lignins, and lignans. These substances protect plants against biotic and abiotic stresses such as pathogenesis, herbivory, wounding, high light/UV, low temperatures, air pollution, and ozone (Christie 1994; Dixon and Paiva 1995; Shirley 1996; Solecka 1997; Sharma et al. 2019). Various stressors alter the redox state (amount of oxidant or antioxidant) which, in turn, serves as a hub to regulate the biosynthesis of antioxidants, phenylpropanoids (Grace and Logan 2000; Foyer and Noctor 2012; Brunetti et al. 2015; Foyer and Noctor 2015; Li et al. 2019a). In bamboo, oxidative stress (exogenous $\mathrm{H}_{2} \mathrm{O}_{2}$ ) activates NADPH oxidase, which induces endogenous $\mathrm{H}_{2} \mathrm{O}_{2}$ accumulation. And then, high level of endogenous $\mathrm{H}_{2} \mathrm{O}_{2}$ accelerates bamboo shoot lignification by activating the phenylpropanoid pathway (Li et al. 2019a). Certain CAD homologs have various enzymatic activities that are unrelated to lignin biosynthesis. However, they are still involved in plant responses to abiotic and biotic stresses (Williamson et al. 1995; Somssich et al. 1996; Li et al. 2019b). In Saccharomyces cerevisiae, CADs may participate in fusel alcohol synthesis, ligninolysis, and $\operatorname{NADP}(\mathrm{H})$ homeostasis (Larroy et al. 2002). The above examples show that CAD functions are not highly conserved among different organisms, but that all CADs appear to be engaged in regulating or responding to changes of the redox status.

Cinnamyl alcohol dehydrogenase (CAD) homologs are ubiquitous in filamentous fungi but their functions remain unclear. Certain recent genomic and metabonomic studies have indicated that the phenylpropanoid pathway may occur in filamentous fungi (Seshime et al. 2005; Zhang et al. 2019b). To the best of our knowledge, however, the presence of the lignin biosynthetic pathway has not yet been demonstrated in filamentous fungi. Hence, CAD homologs are likely to have functions other than lignification in filamentous fungi.

Several studies have identified the molecules and signal transduction pathways involved in the initiation and development of sclerotia in S. sclerotiorum. The accumulation of ROS and lipid peroxidation increases during sclerotial development (Georgiou et al. 2006; Papapostolou and Georgiou 2010), but ROS scavengers have been shown to inhibit sclerotial development in S. sclerotiorum, S. minor, S. rolfsii, and Rhizoctonia solani (Georgiou et al. 2006; Patsoukis and Georgiou 2007, 2008). The present study found that superoxide production was impaired in $S s-C A D$-silenced strains, whereas exogenous oxidants restored sclerotial development. NADPH oxidases are associated with ROS accumulation and regulate sclerotial development in S. sclerotiorum (Kim et al. 2011). NoxR is also a key ROS production factor in many fungi (Takemoto et al. 2006; Scott and Eaton 2008; Tanaka et al. 2008; Hernandez-Onate et al. 2012). The current study found that Nox1, Nox2, and NoxR were downregulated in $S s$-CAD-silenced strains, and demonstrated that $S s-N o x R$ silencing impaired sclerotial formation whereas Ss-Nox1 overexpression in Ss-CADsilenced strains recovered sclerotial development. These results indicate that $S s-C A D$ regulates sclerotial development in S. sclerotiorum via the ROS pathway.

NADPH oxidases play crucial roles in many biological processes, as they use NADPH as a substrate to generate ROS (Ying 2008; Shi et al. 2015). Earlier reports have shown that NADPH homeostasis is essential for cell survival and development (Tan et al. 2009; Jeon et al. 2012; Wang et al. 2014). This study found that NADPH could restore sclerotial development in $S s$-CAD-silenced strains, rather than NADP+ or NADH. Therefore, $\mathrm{NADPH}$ homeostasis was modulated in the $S s$-CAD-silenced strains.

\section{Conclusions}

This study reports that the Ss-CAD protein containing three conserved domains of CAD family is required for sclerotial development in $S$. sclerotiorum, and that NADPH-dependent ROS regulates this process. These findings help to elucidate the functions of CAD in fungi and can be used to develop new strategies of controlling Sclerotinia diseases in plants.

\section{Methods}

\section{Fungal strains and culture conditions}

The wild-type strain Ep-1PNA367 used in this study was a virulent strain of S. sclerotiorum (Xie et al. 2006). It was stored as mycelial plugs on potato dextrose agar (PDA; Difco Laboratories, Detroit, MI, USA) at $4{ }^{\circ} \mathrm{C}$ 
until later use. The strains were routinely sub-cultured on PDA at $20^{\circ} \mathrm{C}$ to maintain vigor and purity. Transformants were cultured on PDA amended with $100 \mathrm{mg} / \mathrm{mL}$ hygromycin B (Calbiochem, San Diego, CA, USA). To facilitate hyphae collection from cultures grown on a solid medium, a sterile cellophane membrane was placed on the agar before inoculation. Sclerotia were produced by incubation at $20^{\circ} \mathrm{C}$ for $30 \mathrm{~d}$ on autoclaved carrot rods in 250 -mL flasks.

\section{Nucleic acid extraction and RT-PCR analysis}

The wild-type strain and transformant mycelial agar disks were inoculated onto PDA overlaid with cellophane and incubated at $20^{\circ} \mathrm{C}$. Hyphae were collected at various time points and were ground to a fine powder in liquid nitrogen. The DNA of the hyphae was extracted following the cetyltrimethylammonium bromide method (Sambrook and Russell 2001). Total RNA was isolated with TRIzol reagent (Huashun Bioengineering Co., Shanghai, China) and was treated with DNaseI (TaKaRa, Dalian, China), according to the manufacturer's instructions. First-strand cDNA was synthesized using RevertAid $^{\text {tw }}$ first-strand cDNA synthesis kits (MBI; Fermentas, Waltham, MA, USA) and this was used as a template for PCR or qRT-PCR.

Relative gene expression quantification in S. sclerotiorum was performed by quantitative real-time PCR with SYBR-Green in a CFX96TM real-time PCR detection system (Bio-Rad Laboratories, Hercules, CA, USA). The corresponding primer pairs were used to determine the expression levels of Ss-CAD, Ss-Nox1, Ss-Nox2, SsNoxR, Ss-SOD, and Ss-CAT (Additional file 3: Table S1). Total cDNA abundance was normalized using actin as a control and was amplified by the actin F/actin $\mathrm{R}$ primer pair (Additional file 3: Table S1).

\section{Construction of RNAi and overexpression vectors}

The Ss-CAD RNAi vectors were constructed according to the methods of Yu et al. (2012). A 528-bp DNA fragment of $S s-C A D$ was amplified by PCR using a specific primer pair. The BamHI and ClaI restriction sites were introduced at the $5^{\prime}$ terminus of the sense primer, and the PstI and HindIII restriction sites were introduced at the $5^{\prime}$ terminus of the antisense primer (Additional file 3: Table S1). The PCR product was co-digested either with BamHI plus PstI or with ClaI plus HindIII to generate DNA fragments with two different types of sticky ends. The fragment pairs were ligated to PCIT and produced a new vector containing a reverse repeat structure separated by a 420 -bp intron. This vector was digested with $\mathrm{XhoI}$ plus $\mathrm{SacI}$ to obtain a repeat fragment and was ligated with the pCAMBIA3300 vector to form the $\mathrm{pSi}$ CAD Ss-CAD-silencing vector. The pSiNoxR Ss-NoxR-silencing vector was also obtained using the aforementioned procedure. Then, $\mathrm{pSiCAD}$ and $\mathrm{pSiNoxR}$ were separately transformed into Agrobacterium tumefaciens EHA105. To construct the Ss-Nox1 overexpression vector, full-length Ss-Nox1 cDNA was amplified using specific primers (Additional file 3: Table S1). The PCR product was ligated with nucleotide A which, in turn, was ligated into the $\mathrm{XcmI}$-digested $\mathrm{pCXH}$ vector.

\section{S. sclerotiorum transformation}

S. sclerotiorum was subjected to Agrobacterium-mediated transformation (Yu et al. 2012), with minor modifications. Fresh A. tumefaciens EHA105 cells containing pSiCAD, pSiNoxR, or pOENox1 were co-cultivated with fresh $S$. sclerotiorum mycelial plugs on a co-induction agar medium overlaid with cellophane. After cocultivation at $20^{\circ} \mathrm{C}$ for $2 \mathrm{~d}$, the plates were covered with selective medium (PDA amended with $100 \mu \mathrm{g} / \mathrm{mL}$ hygromycin $B$ and $200 \mu \mathrm{g} / \mathrm{mL}$ cefotaxime sodium) and were incubated at $20^{\circ} \mathrm{C}$ for $4 \mathrm{~d}$. Colonies regenerated via the selective medium were transferred to PDA containing $100 \mu \mathrm{g} / \mathrm{mL}$ hygromycin B. Transformants were subcultured at least 3 times by transferring the hyphal tips to fresh PDA containing $100 \mu \mathrm{g} / \mathrm{mL}$ hygromycin B.

\section{Exogenous $\mathrm{H}_{2} \mathrm{O}_{2}$ and NADPH treatment}

To determine whether $\mathrm{H}_{2} \mathrm{O}_{2}$ and NADPH could recover sclerotial development in the mutants, the Ep-1PNA367 and SiCAD-51 S. sclerotiorum strains were inoculated onto PDA amended with $6 \mathrm{mM} \mathrm{H}_{2} \mathrm{O}_{2}$ or $0.2 \mathrm{mM}$ $\mathrm{NADPH}$. The controls comprised the aforementioned strains cultured on untreated PDA. Colony morphology, sclerotial development, and sclerotia weight were evaluated for each treatment.

\section{Reactive oxygen species detection assays}

Nitro blue tetrazolium (NBT) staining was used to detect superoxide in the various $S$. sclerotiorum strains as described by Kim et al. (2011). Fungal tissues cultured for 3-4 d on PDA overlaid with sterile cellophane membrane were incubated in $0.5 \mathrm{mg} / \mathrm{mL}$ NBT in $10 \mathrm{mM}$ potassium phosphate buffer ( $\mathrm{pH}$ 7.5) for $2 \mathrm{~h}$. After staining, the fungal tissues were observed by light microscopy.

\section{Enzyme activity assays}

Total proteins were extracted as described by Zhang et al. (2006), with slight modifications. Mycelial agar discs of the wild-type and transformant strains were inoculated onto cellophane-overlaid PDA and were incubated at $20^{\circ} \mathrm{C}$. Fungal hyphae were collected at 48 hours post-inoculation (hpi), ground to a fine powder in liquid nitrogen, and extracted using $1000 \mu \mathrm{L}$ extraction buffer $(100 \mathrm{mM}$ Tris- $\mathrm{HCl}$ (pH 7.5), 2\% polyethylene glycol 6000, $5 \mathrm{mM}$ dithiothreitol, and $2 \%$ polyvinylpolypyrrolidone) for $2.5 \mathrm{~h}$ at $4{ }^{\circ} \mathrm{C}$. The suspension was centrifuged 
at $8000 \mathrm{rpm}$ at $4{ }^{\circ} \mathrm{C}$ for $10 \mathrm{~min}$ and the supernatant was decanted. Then, $100 \mu \mathrm{g}$ total protein was used in CAD activity assays. The protein concentrations were determined by Bradford assays. Assays of the crude enzyme were conducted using coniferyl alcohol as the substrate. Hydroxycinnamaldehyde formation was spectrophotometrically determined at $400 \mathrm{~nm}$ using a coniferaldehyde molar extinction coefficient of $2.10 \times 10^{4} \mathrm{M}^{-1} \mathrm{~cm}^{-1}$ (Sibout et al. 2003). Assays were conducted at $30^{\circ} \mathrm{C}$ for 10 $\min$ in $500 \mu \mathrm{L}$ of $100 \mathrm{mM}$ Tris- $\mathrm{HCl}(\mathrm{pH} \mathrm{8.8)}, 100 \mu \mathrm{M}$ NADP, and $250 \mu \mathrm{M}$ coniferyl alcohol. Enzyme reactions were initiated by enzyme addition and were stopped by incubation at $85^{\circ} \mathrm{C}$ for $10 \mathrm{~min}$. The control consisted of an assay lacking NADP.

\section{Bioinformatics analysis}

The full-length Ss-CAD protein and homolog sequences were retrieved from the NCBI GenBank database (http:// www.ncbi.nlm.nih.gov/genomes) for use in the analyses. The sequence motifs were discovered and analyzed using MEME SUITE.

\section{Observation of sclerotial development}

To observe sclerotial development, all strains were incubated on PDA at $20^{\circ} \mathrm{C}$ for $15 \mathrm{~d}$, and photographs were taken at 6 and $10 \mathrm{dpi}$. The superoxide accumulation levels in the $S s-C A D$-silenced transformants and the wild-type strain were detected by NBT staining after 3-4 dpi onto PDA. Fluorescence intensity was monitored under a light microscope. To determine the effects of exogenous $\mathrm{H}_{2} \mathrm{O}_{2}$ and NADPH on sclerotial development, all strains were incubated on PDA amended either with $6 \mathrm{mM} \mathrm{H}_{2} \mathrm{O}_{2}$ or with $0.2 \mathrm{mM} \mathrm{NADPH}$, and photographs were acquired at $10 \mathrm{dpi}$. All sclerotia were collected, air-dried, and weighed at 15 dpi. The ability of $S s$-CAD-silenced strains to produce sclerotia on carrots was also determined. SiCAD-6, SiCAD-10, and SiCAD-5 and the corresponding wild-type strain were incubated on $100 \mathrm{~g}$ autoclaved carrot rods in 250-mL flasks at $20^{\circ} \mathrm{C}$ for $30 \mathrm{~d}$ and photographs were taken at $10 \mathrm{dpi}$ and $30 \mathrm{dpi}$. The sclerotia were washed and dried at $28^{\circ} \mathrm{C}$. For each strain, 100 randomly selected sclerotia were photographed and weighed. Three independent replicates were performed for all experiments.

\section{Morphological observations and pathogenicity assays}

To observe colonial morphology, all strains were incubated on PDA at $20^{\circ} \mathrm{C}$ under a $12 \mathrm{~h}$ light $/ 12 \mathrm{~h}$ dark photoperiod. After $1 \mathrm{~d}$ incubation, hyphal development was observed and photographed under a dissecting microscope. The colony diameters of the wild-type and Ss-CAD-silenced strains were measured at $12 \mathrm{hpi}$ and 36 hpi. The vegetative growth rate of each strain was calculated as follows: growth rate $(\mathrm{cm} / \mathrm{d})=(36 \mathrm{hpi}$ diameter -
12 hpi diameter). Three independent replicates were performed per experiment.

The virulence of the $S s$-CAD-silenced transformants was tested using detached healthy rapeseed leaves. Mycelial plugs $6 \mathrm{~mm}$ in diameter were excised from the margins of active fungal colonies on PDA, placed inverted on detached leaves, and incubated in a plastic chamber at $95 \% \mathrm{RH}$ and $20^{\circ} \mathrm{C}$. To evaluate disease severity, lesions were measured at $48 \mathrm{hpi}$. The wild-type strain served as the control. Five leaves were inoculated per strain and three independent replicates were performed.

\section{Supplementary information}

Supplementary information accompanies this paper at https://doi.org/10. 1186/s42483-020-00056-9.

Additional file 1: Figure S1. Three conserved motifs were derived from Ss-CAD and its homologs in Arabidopsis thaliana and Saccharomyces cerevisiae by the MEME tool. Height of each letter represents relative frequency of each base at various positions in the consensus sequence. a Zn1 binding motif $\left[\mathrm{GHE}(X)_{2} \mathrm{G}(X)_{5} \mathrm{G}(X)_{2} \mathrm{~V}\right]$. b Structural Zn2 binding motif $\left[G D(X)_{9.10} C(X)_{2} C(X)_{2} C(X)_{7} C\right]$. c NADPH binding motif $[G(X) G G V(L) G]$.

Additional file 2: Figure S2. Influence of SS-CAD silencing on Sclerotia sclerotiorum growth rate and pathogenicity. a Comparison of growth rates of Ss-CAD-silenced transformants and the wild-type strain. b Ss-CADsilenced transformants showing unchanged virulence on detached rapeseed (Brassica napus) leaf. Photographs were taken at 48 hpi. c Comparison of diameters of lesions induced by Ss-CAD-silenced transformants against those induced by the wild-type strain. Three independent replicates were performed per experiment. Values are means \pm S.E. ${ }^{*} P<0.05$; one-way ANOVA.

Additional file 3: Table S1. Primers used in the present study.

\section{Abbreviations}

CAD: Cinnamyl alcohol dehydrogenase; NBT: Nitro blue tetrazolium; qRTPCR: Quantitative real-time reverse-transcription PCR; ROS: Reactive oxygen species

\section{Acknowledgments}

Not applicable.

Authors' contributions

$J Z$ and JC conceived and designed the research. JZ, YL, YF, JX, and DJ performed the experiments and analyzed the data. JZ, YL, and JC wrote the manuscript. All authors read and approved the final manuscript.

\section{Funding}

This work was financially supported by the National Key Research and Development Program of China (No. 2018YFD0200900) and the earmarked fund for the China Agriculture Research System (No. CARS-13).

Availability of data and materials

Not applicable.

Ethics approval and consent to participate Not applicable.

Consent for publication

Not applicable.

Competing interests

The authors declare that they have no competing interests. 
Received: 28 March 2020 Accepted: 28 May 2020

Published online: 08 June 2020

\section{References}

Adams PB, Ayers WA. Ecology of Sclerotinia species. Phytopathology. 1979;69: 896-9.

Bailey TL, Boden M, Buske FA, Frith M, Grant CE, Clementi L, et al. MEME SUITE: tools for motif discovery and searching. Nucleic Acids Res. 2009;37:W202-8.

Boerjan W, Ralph J, Baucher M. Lignin biosynthesis. Annu Rev Plant Biol. 2003:54: 519-46.

Boland GJ, Hall R. Index of plant hosts of Sclerotinia sclerotiorum. Can J Plant Pathol. 1994;16:93-100.

Bolton MD, Thomma BPHJ, Nelson BD. Sclerotinia sclerotiorum (lib.) de Bary: biology and molecular traits of a cosmopolitan pathogen. Mol Plant Pathol. 2006;7:1-16.

Brunetti C, Guidi L, Sebastiani F, Tattini M. Isoprenoids and phenylpropanoids are key components of the antioxidant defense system of plants facing severe excess light stress. Environ Exp Bot. 2015;119:54-62.

Chen C, Dickman MB. CAMP blocks MAPK activation and sclerotial development via Rap-1 in a PKA-independent manner in Sclerotinia sclerotiorum. Mol Microbiol. 2005;55:299-311.

Chen C, Harel A, Gorovoits R, Yarden O, Dickman MB. MAPK regulation of sclerotial development in Sclerotinia sclerotiorum is linked with $\mathrm{pH}$ and CAMP sensing. Mol Plant-Microbe Interact. 2004:17:404-13.

Chet I, Henis Y. Sclerotial morphogenesis in fungi. Annu Rev Phytopathol. 1975; 13:169-92.

Christie PJ. Impact of low-temperature stress on general phenylpropanoid and anthocyanin pathways: enhancement of transcript abundance and anthocyanin pigmentation in maize seedlings. Planta. 1994;194:541-9.

Dixon RA, Paiva NL. Stress-induced phenylpropanoid metabolism. Plant Cell. 1995;7:1085-97.

Ebel J, Grisebach H. Reduction of cinnamic acids to cinnamyl alcohols with an enzyme preparation from cell suspension cultures of soybean (Glycine max) FEBS Lett. 1973;30:141-3.

Erental A, Harel A, Yarden O. Type 2A phosphoprotein phosphatase is required for asexual development and pathogenesis of Sclerotinia sclerotiorum. Mol Plant-Microbe Interact. 2007;20:944-54.

Fan H, Yu G, Liu Y, Zhang X, Liu J, Zhang Y, et al. An atypical forkhead-containing transcription factor SsFKH1 is involved in sclerotial formation and is essential for pathogenicity in Sclerotinia sclerotiorum. Mol Plant Pathol. 2017;18:963-75.

Foyer $\mathrm{CH}$, Noctor G. Managing the cellular redox hub in photosynthetic organisms. Plant Cell Environ. 2012;35:199-201.

Foyer $\mathrm{CH}$, Noctor $\mathrm{G}$. Defining robust redox signalling within the context of the plant cell. Plant Cell Environ. 2015:38:239.

Georgiou CD, Patsoukis N, Papapostolou I, Zervoudakis G. Sclerotial metamorphosis in filamentous fungi is induced by oxidative stress. Integr Comp Biol. 2006:46:691-712

Grace SC, Logan BA. Energy dissipation and radical scavenging by the plant phenylpropanoid pathway. Philos Trans R Soc Lond Ser B Biol Sci. 2000;355:1499-510.

Harel A, Bercovich S, Yarden O. Calcineurin is required for sclerotial development and pathogenicity of Sclerotinia sclerotiorum in an oxalic acid-independent manner. Mol Plant-Microbe Interact. 2006:19:682-93.

Hegedus DD, Rimmer SR. Sclerotinia sclerotiorum: when "to be or not to be" a pathogen? FEMS Microbiol Lett. 2005;251:177-84

Hernandez-Onate MA, Esquivel-Naranjo EU, Mendoza-Mendoza A, Stewart A, Herrera-Estrella $\mathrm{AH}$. An injury-response mechanism conserved across kingdoms determines entry of the fungus Trichoderma atroviride into development. Proc Natl Acad Sci U S A. 2012;109:14918-23.

Jeon SM, Chandel NS, Hay N. AMPK regulates NADPH homeostasis to promote tumour cell survival during energy stress. Nature. 2012;485:661-5.

Jörnvall $\mathrm{H}$, Jo H. Nomenclature of alcohol dehydrogenases. Alcohol Alcohol. 1995;30:153-61.

Jurick WM, Dickman MB, Rollins JA. Characterization and functional analysis of a cAMP-dependent protein kinase a catalytic subunit gene (pka1) in Sclerotinia sclerotiorum. Physiol Mol Plant Pathol. 2004;64:155-63.

Jurick WM, Rollins JA. Deletion of the adenylate cyclase (sac1) gene affects multiple developmental pathways and pathogenicity in Sclerotinia sclerotiorum. Fungal Genet Biol. 2007;44:521-30.

Kim HJ, Chen C, Kabbage M, Dickman MB. Identification and characterization of Sclerotinia sclerotiorum NADPH oxidases. Appl Environ Microbiol. 2011;77: 7721-9.
Kim SJ, Kim MR, Bedgar DL, Moinuddin SG, Cardenas CL, Davin LB, et al. Functional reclassification of the putative cinnamyl alcohol dehydrogenase multigene family in Arabidopsis. Proc Natl Acad Sci U S A. 2004;101:1455-60.

Larroy C, Pares X, Biosca JA. Characterization of a Saccharomyces cerevisiae $\mathrm{NADP}(\mathrm{H})$-dependent alcohol dehydrogenase (ADHVII), a member of the cinnamyl alcohol dehydrogenase family. Eur J Biochem. 2002;269:5738-45.

Li D, Limwachiranon J, Li L, Zhang L, Xu Y, Fu M, et al. Hydrogen peroxide accelerated the lignification process of bamboo shoots by activating the phenylpropanoid pathway and programmed cell death in postharvest storage. Postharvest Biol Technol. 2019a;153:79-86.

Li D, Qin X, Tian P, Wang J. Toughening and its association with the postharvest quality of king oyster mushroom (Pleurotus eryngii) stored at low temperature. Food Chem. 2016;196:1092-100.

Li J, Mu W, Veluchamy S, Liu Y, Zhang Y, Pan H, et al. The GATA-type IVb zinc-finger transcription factor SsNsd1 regulates asexual-sexual development and appressoria formation in Sclerotinia sclerotiorum. Mol Plant Pathol. 2018;19:1679-89.

Li M, Rollins JA. The development-specific protein (Ssp1) from Sclerotinia sclerotiorum is encoded by a novel gene expressed exclusively in sclerotium tissues. Mycologia. 2009;101:34-43.

Li M, Rollins JA. The development-specific ssp1 and ssp2 genes of Sclerotinia sclerotiorum encode lectins with distinct yet compensatory regulation. Fungal Genet Biol. 2010;47:531-8.

Li T, Wang Q, Feng R, Li L, Ding L, Fan G, et al. Negative regulators of plant immunity derived from cinnamyl alcohol dehydrogenases are targeted by multiple Phytophthora Avr3a-like effectors. New Phytol. 2019b. https://doi. org/10.1111/nph.16139.

Liang Y, Xiong W, Steinkellner S, Feng J. Deficiency of the melanin biosynthesis genes SCD1 and THR1 affects sclerotial development and vegetative growth, but not pathogenicity, in Sclerotinia sclerotiorum. Mol Plant Pathol. 2018;19: 1444-53.

Lyu X, Shen C, Fu Y, Xie J, Jiang D, Li G, et al. Comparative genomic and transcriptional analyses of the carbohydrate-active enzymes and secretomes of phytopathogenic fungi reveal their significant roles during infection and development. Sci Rep. 2015;5:15565.

Lyu X, Shen C, Fu Y, Xie J, Jiang D, Li G, et al. The microbial opsin homolog Sop1 is involved in Sclerotinia sclerotiorum development and environmental stress response. Front Microbiol. 2016;6:1504.

McKie JH, Jaouhari R, Douglas KT, Goffner D, Feuillet C, Grima-Pettenati J, et al. A molecular model for cinnamyl alcohol dehydrogenase, a plant aromatic alcohol dehydrogenase involved in lignification. Biochim Biophys Acta. 1993: 1202:61-9.

Papapostolou I, Georgiou CD. Superoxide radical is involved in the sclerotial differentiation of filamentous phytopathogenic fungi: identification of a fungal xanthine oxidase. Fungal Biol. 2010;114:387-95.

Patsoukis N, Georgiou CD. Effect of thiol redox state modulators on oxidative stress and sclerotial differentiation of the phytopathogenic fungus Rhizoctonia solani. Arch Microbiol. 2007;188:225-33.

Patsoukis N, Georgiou CD. Differentiation of Sclerotinia minor depends on thiol redox state and oxidative stress. Can J Microbiol. 2008;54:28-36.

Preisner M, Kulma A, Zebrowski J, Dyminska L, Hanuza J, Arendt M, et al. Manipulating cinnamyl alcohol dehydrogenase (CAD) expression in flax affects fibre composition and properties. BMC Plant Biol. 2014;14:50.

Roper M, Seminara A, Bandi MM, Cobb A, Dillard HR, Pringle A. Dispersal of fungal spores on a cooperatively generated wind. Proc Natl Acad Sci U S A. 2010;107:17474-9.

Rossmann MG, Moras D, Olsen KW. Chemical and biological evolution of nucleotide-binding protein. Nature. 1974;250:194-9.

Saballos A, Ejeta G, Sanchez E, Kang C, Vermerris W. A genomewide analysis of the cinnamyl alcohol dehydrogenase family in sorghum [Sorghum bicolor (L.) Moench] identifies SbCAD2 as the brown midrib6 gene. Genetics. 2009;181:783-95.

Sambrook J, Russell DW. Molecular cloning: a laboratory manual (3rd edition). New York: Cold Spring Harbor Laboratory; 2001.

Scott B, Eaton CJ. Role of reactive oxygen species in fungal cellular differentiations. Curr Opin Microbiol. 2008;11:488-93.

Seo KH, Zhuang N, Chen C, Song JY, Kang HL, Rhee KH, et al. Unusual NADPH conformation in the crystal structure of a cinnamyl alcohol dehydrogenase from Helicobacter pylori in complex with NADP(H) and substrate docking analysis. FEBS Lett. 2012;586:337-43.

Seshime Y, Juvvadi PR, Fujii I, Kitamoto K. Genomic evidences for the existence of a phenylpropanoid metabolic pathway in Aspergillus oryzae. Biochem Biophys Res Commun. 2005;337:747-51. 
Sharma A, Shahzad B, Rehman A, Bhardwaj R, Landi M, Zheng B. Response of phenylpropanoid pathway and the role of polyphenols in plants under abiotic stress. Molecules. 2019;24:2452.

Shi L, Gong L, Zhang X, Ren A, Gao T, Zhao M. The regulation of methyl jasmonate on hyphal branching and GA biosynthesis in Ganoderma lucidum partly via ROS generated by NADPH oxidase. Fungal Genet Biol. 2015;81:201-11.

Shirley BW. Flavonoid biosynthesis: 'New' functions for an 'old' pathway. Trends Plant Sci. 1996;1:377-82.

Sibout R, Eudes A, Mouille G, Pollet B, Lapierre C, Jouanin L, et al. CINNAMYL ALCOHOL DEHYDROGENASE- $C$ and $-D$ are the primary genes involved in lignin biosynthesis in the floral stem of Arabidopsis. Plant Cell. 2005;17:2059-76.

Sibout R, Eudes A, Pollet B, Goujon T, Mila I, Granier F, et al. Expression pattern of two paralogs encoding cinnamyl alcohol dehydrogenases in Arabidopsis. Isolation and characterization of the corresponding mutants. Plant Physiol. 2003:132:848-60

Solecka D. Role of phenylpropanoid compounds in plant responses to different stress factors. Acta Physiol Plant. 1997;19:257-68.

Somssich IE, Wernert P, Kiedrowski S, Hahlbrock K. Arabidopsis thaliana defenserelated protein ELI3 is an aromatic alcohol: NADP+ oxidoreductase. Proc Natl Acad Sci U S A. 1996;93:14199-203.

Steadman JR. Control of plant diseases caused by Sclerotinia species. Phytopathology. 1979;69:904-7.

Takemoto D, Tanaka A, Scott B. A p67(Phox)-like regulator is recruited to control hyphal branching in a fungal-grass mutualistic symbiosis. Plant Cell. 2006;18:2807-21.

Tan SX, Teo M, Lam YT, Dawes IW, Perrone GG. Cu, Zn superoxide dismutase and $\operatorname{NADP}(H)$ homeostasis are required for tolerance of endoplasmic reticulum stress in Saccharomyces cerevisiae. Mol Biol Cell. 2009;20:1493-508.

Tanaka A, Takemoto D, Hyon GS, Park P, Scott B. NoxA activation by the small GTPase RacA is required to maintain a mutualistic symbiotic association between Epichloe festucae and perennial nyegrass. Mol Microbiol. 2008;68:1165-78.

Tobias CM, Chow EK. Structure of the cinnamyl-alcohol dehydrogenase gene family in rice and promoter activity of a member associated with lignification. Planta. 2005;220:678-88.

Valencia E, Larroy C, Ochoa WF, Pares X, Fita I, Biosca JA. Apo and Holo structures of an NADPH-dependent cinnamyl alcohol dehydrogenase from Saccharomyces cerevisiae. J Mol Biol. 2004;341:1049-62.

Wang YP, Zhou LS, Zhao YZ, Wang SW, Chen LL, Liu LX, et al. Regulation of G6PD acetylation by SIRT2 and KAT9 modulates NADPH homeostasis and cell survival during oxidative stress. EMBO J. 2014;33:1304-20.

Wilkin JM, Soetaert K, Stelandre M, Buyssens P, Castillo G, Demoulin V, et al. Overexpression, purification and characterization of Mycobacterium bovis BCG alcohol dehydrogenase. Eur J Biochem. 1999;262:299-307.

Williamson JD, Stoop JM, Massel MO, Conkling MA, Pharr DM. Sequence analysis of a mannitol dehydrogenase CDNA from plants reveals a function for the pathogenesis-related protein ELI3. Proc Natl Acad Sci U S A. 1995;92:7148-52.

Xiao X, Cheng J, Tang J, Fu Y, Jiang D, Baker TS, et al. A novel partitivirus that confers hypovirulence on plant pathogenic fungi. J Virol. 2014;88:10120-33.

Xie J, Wei D, Jiang D, Fu Y, Li G, Ghabrial S, et al. Characterization of debilitationassociated mycovirus infecting the plant-pathogenic fungus Sclerotinia sclerotiorum. J Gen Virol. 2006:87:241-9.

Ying W. NAD+/NADH and NADP+/NADPH in cellular functions and cell death: regulation and biological consequences. Antioxid Redox Signal. 2008;10:179-206.

Yu Y, Jiang D, Xie J, Cheng J, Li G, Yi X, et al. Ss-SI2, a novel cell wall protein with PAN modules, is essential for sclerotial development and cellular integrity of Sclerotinia sclerotiorum. PLoS One. 2012;7:e34962.

Zhang J, Wang Y, Du J, Huang Z, Fang A, Yang Y, et al. Sclerotinia sclerotiorum thioredoxin reductase is required for oxidative stress tolerance, virulence, and sclerotial development. Front Microbiol. 2019a;10:233.

Zhang K, Qian Q, Huang Z, Wang Y, Li M, Hong L, et al. GOLD HULL AND INTERNODE2 encodes a primarily multifunctional cinnamyl-alcohol dehydrogenase in rice. Plant Physiol. 2006:140:972-83.

Zhang Y, Shi J, Ni Y, Liu Y, Zhao Z, Zhao X, et al. Tracing the mass flow from glucose and phenylalanine to pinoresinol and its glycosides in Phomopsis sp. XP-8 using stable isotope assisted TOF-MS. Sci Rep. 2019b;9:18495.

Zhu W, Wei W, Fu Y, Cheng J, Xie J, Li G, et al. A secretory protein of necrotrophic fungus Sclerotinia sclerotiorum that suppresses host resistance. PLoS One. 2013;8:e53901.

\section{Ready to submit your research? Choose BMC and benefit from:}

- fast, convenient online submission

- thorough peer review by experienced researchers in your field

- rapid publication on acceptance

- support for research data, including large and complex data types

- gold Open Access which fosters wider collaboration and increased citations

- maximum visibility for your research: over $100 \mathrm{M}$ website views per year

At BMC, research is always in progress.

Learn more biomedcentral.com/submissions 